\title{
Epidemiology and Recurrence Rates of Clostridium difficile Infections in Germany: A Secondary Data Analysis
}

Christoph Lübbert $\cdot$ Lisa Zimmermann · Julia Borchert ·

Bernd Hörner · Reinier Mutters · Arne C. Rodloff

Received: September 15, 2016 / Published online: October 21, 2016

(C) The Author(s) 2016. This article is published with open access at Springerlink.com

\begin{abstract}
Introduction: Clostridium difficile infection (CDI) is the most common cause of health-care-associated infectious diarrhea. Recurrence rates are as high as $20-30 \%$ after standard treatment with metronidazole or vancomycin, and appear to be reduced for patients treated with fidaxomicin. According to the literature, the risk of CDI recurrence
\end{abstract}

Christoph Lübbert and Lisa Zimmermann have contributed equally to this work.

Enhanced content To view enhanced content for this article go to http://www.medengine.com/Redeem/ 6F07F0602CB8ADAE.

C. Lübbert $(\bowtie)$

Division of Infectious Diseases and Tropical

Medicine, Department of Gastroenterology and

Rheumatology, Leipzig University Hospital,

Liebigstr. 20, 04103 Leipzig, Germany

e-mail: christoph.luebbert@medizin.uni-leipzig.de

C. Lübbert · A. C. Rodloff

Interdisciplinary Center for Infectious Diseases,

Leipzig University Hospital, 04103 Leipzig,

Germany

L. Zimmermann · J. Borchert

Gesundheitsforen Leipzig GmbH, 04109 Leipzig,

Germany increases after the second relapse to 30-65\%. Accurate data for Germany are not yet available. Methods: Based on the research database of arvato health analytics (Munich, Germany), a secondary data analysis for the incidence, treatment characteristics and course of CDI was performed. The database included high granular accounting information of about 1.46 million medically insured patients covering the period 2006-2013, being representative for Germany. The analysis was based on new-onset CDI in 2012 in patients which either received outpatient antibiotic therapy for CDI or were hospitalized.

Results: The ICD-10 coded incidence of CDI in 2012 was 83 cases per 100,000 population.

B. Hörner

MSD Sharp \& Dohme GmbH, 85540 Haar, Germany

R. Mutters

Institute of Medical Microbiology and Hygiene, Philipps University Marburg, 35043 Marburg, Germany

A. C. Rodloff

Institute for Medical Microbiology and

Epidemiology of Infectious Diseases, Leipzig

University Hospital, 04103 Leipzig, Germany 
Overall mortality rates within the follow-up period of 1 year were $13.5 \%$ in inpatients with primary diagnosis of CDI, compared to $24.3 \%$ in inpatients with secondary diagnosis of CDI $(P<0.001), \quad$ and $\quad 7.1 \% \quad$ in outpatients $(P<0.001)$. In the median, patients with secondary diagnosis of CDI remained significantly longer hospitalized (24 vs. 9 days, $P<0.001)$. First recurrence of CDI was observed in $18.2 \%$ of cases with index events. There was a significantly increased risk to suffer a second and third recurrence, reaching $28.4 \%$ $(P<0.001), \quad$ and $\quad 30.2 \% \quad(P=0.017)$, respectively. Antibiotic therapy of CDI in outpatients was performed mainly with metronidazole (in $90.8 \%$ of index events, $60.0 \%$ of first recurrences, and $43.5 \%$ of second recurrences).

Conclusion: The reported incidence of CDI in Germany is higher than noted previously. The recurrence rates do increase with the number of relapses, but are lower than reported in the literature, despite dominance of metronidazole treatment in outpatients.

Funding: MSD Sharp \& Dohme GmbH, Haar, Germany.

Keywords: Antibiotic treatment; Clostridium difficile infection; Germany; Incidence; Recurrence; Societal burden

\section{INTRODUCTION}

Clostridium difficile infection (CDI) is the most common cause of health-care-associated infectious diarrhea, affecting primarily elderly patients (>65 years) with comorbidities and exposure to antibiotics [1-5]. At least $7-17 \%$ of adult hospitalized patients are colonized by $C$. difficile, with higher rates observed in elderly long-term patients $[4,6]$. C. difficile is also responsible for diarrheal diseases in patients with no risk factors (community-acquired CDI) $[1,5,7]$, and is associated with zoonotic transmission, particularly PCR-ribotype 078 $[8,9]$. Highly virulent $C$. difficile strains have emerged since 2003 leading to a predominance of PCR-ribotype 027 in many hospitals of North America and Europe. This development is made responsible for increased severity of illness and increased mortality $[1-5,7,10,11]$. In Canada, a cumulative attributable mortality of $16.7 \%$ was demonstrated for CDI patients after the arrival of PCR-ribotype 027 [11]. Each case of nosocomial CDI led, on average, to 10.7 additional days in hospital [11]. In a Dutch case-control study, the highest mortality was also seen among very elderly patients and patients with PCR-ribotype 027 [12]. The occurrence of CDI was associated with a 2.5-fold increase in 30-day mortality compared to controls without diarrhea when adjusted for age, sex, and underlying diseases. CDI-related death occurred mainly within 30 days after diagnosis [12].

Recurrence rates of CDI given in the literature are as high as $20-30 \%$ after standard treatment with metronidazole or vancomycin [13], and appear to be reduced for patients treated with fidaxomicin [14-16], which became available for treatment of CDI in Germany at the end of 2012. According to Surawicz and other authors, the risk of CDI recurrence increases after the second relapse to $30-65 \%[17,18]$. The current overall economic burden per CDI, addressed as direct treatment costs, has been reported to reach between $€ 7147$ and $€ 22,800$ [19-21]. However, CDI recurrence is particularly associated with excessive costs, which are mostly attributable to a significantly longer overall length of hospital stay [22]. In a recent German study, direct treatment costs per patient differed significantly: $€ 18,460$ in CDI patients without recurrence, $€ 73,900$ in patients 
with recurrent $\mathrm{CDI}$, and $€ 14,530$ in controls $(P<0.001)$ [22]. The high prevalence of CDI itself also contributes to the economic burden. According to the German Federal Office of Statistics (Statistisches Bundesamt, Wiesbaden, Germany), there is a steadily increasing number of CDI cases (ICD-10 code A04.7) reaching more than 30,000 primary inpatient cases and more than 74,000 secondary inpatient cases in the years 2013 and 2014 in Germany [23].

This paper aims to describe the current incidence, treatment characteristics and recurrence rates of CDI in Germany, derived from the research database of arvato health analytics GmbH (Munich, Germany). The database is run by arvato in cooperation with Gesundheitsforen Leipzig $\mathrm{GmbH}$ (Leipzig, Germany), both offering independent knowledge services and aiming to establish a statistically valid database for health research and analysis. Different German statutory health insurances (voluntary development partners) annually provide their accounting information for this database allowing to analyze high granular health data and insurance biographies over several years. With coverage of patients from all parts of the country, the population investigated seems to be fairly representative for the whole German population.

\section{METHODS}

\section{Study Population and Data Analysis}

Based on the research database of arvato health analytics (Munich, Germany), a secondary data analysis for the incidence, recurrence and course of CDI was performed. The database used for this purpose included high granular accounting information of about 1.46 million medically insured patients covering the period
2006-2013. The analysis was based on all new-onset CDI found between 1 January 2012 and 31 December 2012 in patients who either received outpatient antibiotic treatment for CDI or were hospitalized. CDI-related medication included metronidazole, vancomycin or fidaxomicin, to verify outpatient encoding, but data on medication were not available for inpatients. The index dates were defined by (1) the antibiotic prescription date as a surrogate for outpatient CDI diagnoses, (2) the day of admission for primary inpatient CDI diagnoses, or (3) the date of discharge from the hospital minus 10 days (but no less than the day of admission) for secondary inpatient CDI diagnoses. The latter definition tries to address the problem of hospital-acquired CDI at an unknown date within the hospitalization period considering that the standard length of antibiotic therapy for CDI is 10 days.

New-onset cases were defined as patients with CDI documentation according to ICD-10 diagnosis A04.7 in the database, but with no encodings for CDI or supply with a CDI-related medication for a period of at least 60 days prior to the diagnosis. Since inpatient diagnoses were available for the exact date of hospital admission, and outpatient diagnoses were documented only quarterly due to different accounting systems, separate patient selection algorithms had to be applied. For inpatient diagnoses, the ICD codes of discharge information were used. Another criterion was the absence of CDI-related medication (metronidazole, vancomycin, or fidaxomicin) within 60 days prior to encoding of ICD-10 diagnosis A04.7.

All CDI patients were followed-up for 1 year from the index event or until death. New CDI cases were meticulously analyzed as to whether and how often a recurrence occurred, defined as re-encoding of the ICD-10 code A04.7, or outpatient diagnosis in conjunction with 
CDI-related medication, within 11-60 days of follow-up. Events within 0-10 days of follow-up were not counted as recurrences, because standard CDI drug therapy extends for 10 days. Thus, encoding of a later CDI event ( $\geq 60$ days) was counted as a new index event. Events during hospitalization were further distinguished according to primary or secondary diagnosis of CDI. Accordingly, the length of hospital stay (LOS) refers to the overall LOS and not the LOS attributed to CDI.

\section{Statistical Analysis}

Statistical analysis was performed using SPSS for Windows (SPSS 20.0; IBM, Armonk, New York, USA). Numerical variables were summarized as median, and categorical variables were given as frequencies or proportions. Categorical data were analyzed by the Chi square test or Fisher's exact test. For comparison of two independent groups, the nonparametric Mann-Whitney $U$ test was used. $P$ values ( 2 -sided) of $<0.05$ were considered statistically significant.

\section{Ethics Compliance}

This retrospective study was performed in accordance with the ethical guidelines of the 1964 Declaration of Helsinki and its later amendments. For this type of study, formal consent is not required in accordance with the federal legislation of the Free State of Saxony, Germany. As the study did not modify patient management and the data were processed anonymously, the need for informed consent was waived.

\section{RESULTS}

In 2012, 1223 new CDI cases were identified from a total of 1,461,268 continuously medically insured patients. This corresponds to 83 reported cases per 100,000 population. An extrapolation of this sample to statutory and private health insurances in Germany (1223 of $1,461,268$ refers to 65,000 in a population of $80,000,000$ ) results in more than 65,000 CDI cases within Germany per year (Table 1 ). The hidden incidence of CDI not treated with CDI-specific therapy is not included in these numbers. Thus, an underestimation has to be assumed.

In the analyzed group of CDI cases, 1039 index events (85.0\%) occurred during hospitalization and 184 index events (15.0\%) occurred among outpatients. A total of $61.2 \%$ of the index events (732 patients) occurred in women, being generally more often affected by CDI than men. Women of $80-84$ years were most frequently affected, with a total of 133 CDI patients (11.1\%). In comparison, there were only 61 male patients $(5.1 \%)$ in the same age group. The age peak in men occurred at 75-79 years (84 patients, $7.0 \%$ ), slightly below that of women. The median LOS in patients with primary diagnosis of CDI was 9 days (interquartile range: 6-13 days). In $55.4 \%$ of the cases, the LOS was up to 9 days. In a further

Table 1 CDI cases identified in the research database in 2012

\begin{tabular}{lcl}
\hline Patient selection & CDI cases $(n)$ & $\begin{array}{l}\text { Extrapolation } \\
(n)\end{array}$ \\
\hline New CDI cases & $1223(0.083 \%)$ & 65,252 \\
Inpatients & $1039(85.0 \%)$ & 55,435 \\
$\quad$ Primary diagnosis & $260(25.0 \%)$ & 13,872 \\
$\quad$ A04.7 & & \\
Secondary diagnosis & $779(75.0 \%)$ & 41,563 \\
$\quad$ A04.7 & & \\
Outpatients & $184(15.0 \%)$ & 9817 \\
\hline
\end{tabular}

An extrapolation of this sample to other statutory and private health insurances in Germany is given in the righthand column 


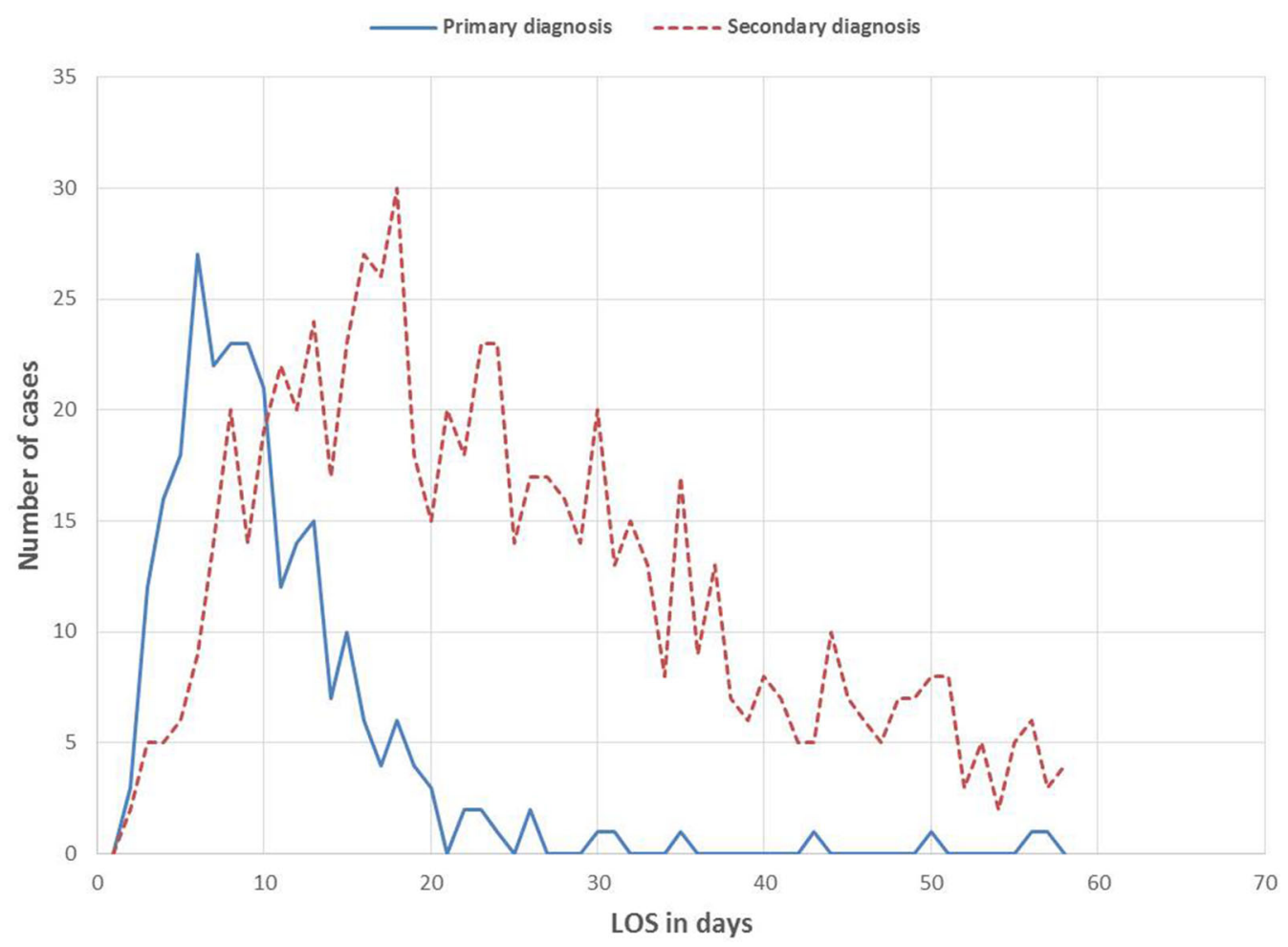

Fig. 1 Overall length of stay in patients ( $L O S$, index events) with either primary or secondary diagnosis of CDI

$38.1 \%$, the LOS varied from 10 to 19 days. Longer hospital stays were rather rare. Patients with a secondary diagnosis of CDI remained significantly longer in the hospital (median 24 days, $P<0.001$ ) (Fig. 1). A total of $13.5 \%$ of inpatients with primary diagnosis of CDI (index events) died within the follow-up period of 1 year, compared to $24.3 \%$ with secondary diagnosis of CDI $(P=0.018)$. By contrast, in the outpatient setting, only $7.1 \%$ of $\mathrm{CDI}$ patients (index events) died within 1 year after the diagnosis was encoded, revealing a significant difference compared with inpatients with primary diagnosis of CDI $(P<0.001)$. The overall mortality rates increased significantly from index event to second recurrence of CDI (Fig. 2).

First recurrence of CDI was observed in $18.2 \%$ of cases with index events (222 of 1223). Referring to first recurrence, there was a significantly increased risk to suffer a second and third recurrence, reaching $28.4 \%$ (63 of $222, P<0.001$ ), and $30.2 \%$ (19 of $63, P=0.017$ ), respectively. In 22 of 53 inpatients with primary diagnosis of CDI (41.5\%), the first recurrence was encoded according to ICD-10 (A04.7) within 11-19 days. In another $15.1 \%$ of cases, recurrence of CDI was documented within 20-29 days. There was no difference regarding the time interval to the occurrence of the first recurrence between inpatients with primary or secondary diagnosis of CDI. For a detailed description of CDI recurrence rates in inpatients and outpatients, see Fig. 3. Details on antibiotic treatment regimens for CDI in outpatients are given in Fig. 4. A high percentage of patients were treated with metronidazole for the first $(60.0 \%)$ and even for the second recurrence (43.5\%). There were no patients treated with fidaxomicin. 


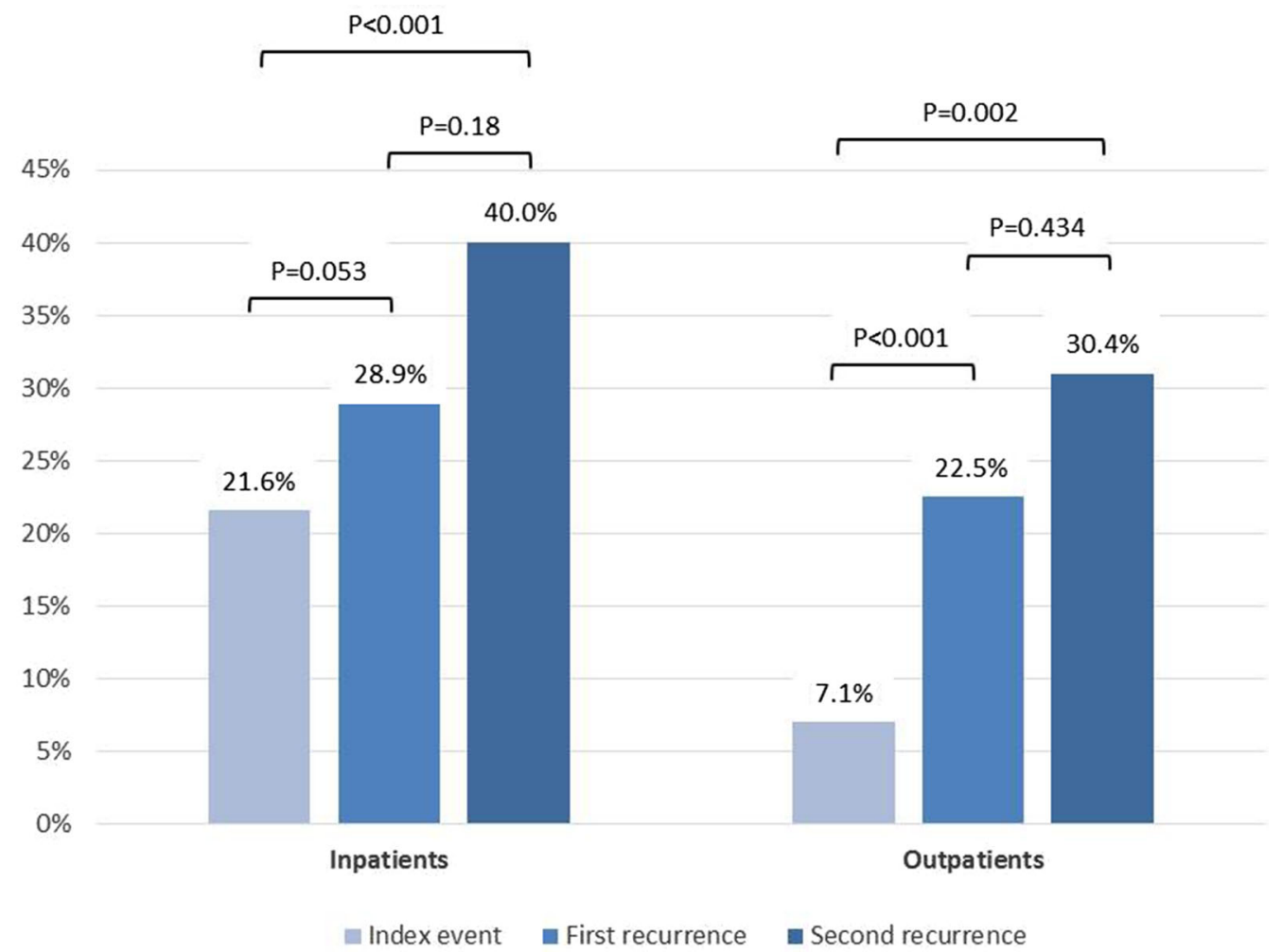

Fig. 2 One-year mortality rates of study patients depending on the recurrence of CDI

\section{DISCUSSION}

According to our research database, the reported incidence of CDI in Germany in 2012 was at least 83 cases per 100,000 population. The prevailing view in the literature clearly shows lower incidences and gives blurred notes on the increasing number of CDI cases in Germany. Burkhardt et al. mention only 14.8 cases per 100,000 population in 2006 in the Federal State of Saxony [24], but Strausberg specifies an estimated incidence of 82 cases per 100,000 population in 2015 referring to the reporting data of the German Institute for the Hospital Remuneration System (Institut für das Entgeltsystem im Krankenhaus, InEK; Siegburg, Germany) [25]. The increased inpatient mortality rates are strongly associated with a high probability of increased comorbidity, especially in patients with CDI as secondary diagnosis.

According to various publications, $20-30 \%$ of CDI patients suffer a recurrence after initial treatment [13-16, 26], which is consistent with our results and shows that this trend is at least as high as previously assumed. According to Surawicz and other authors, the risk of CDI recurrence after the second relapse increases to $30-65 \%[17,18,25]$. This estimate could not be reproduced in our analysis (recurrence of CDI was observed in $18.2 \%$ of the cases, and there was a significant increase in the frequency of a second and third recurrence, reaching 28.4\% and $30.2 \%$, respectively). However, it was generally confirmed that there is a steadily increasing risk after each relapse for ongoing recurrence, which is associated with a significant increase in overall mortality 


\section{Outpatients with diagnosis of CDI}

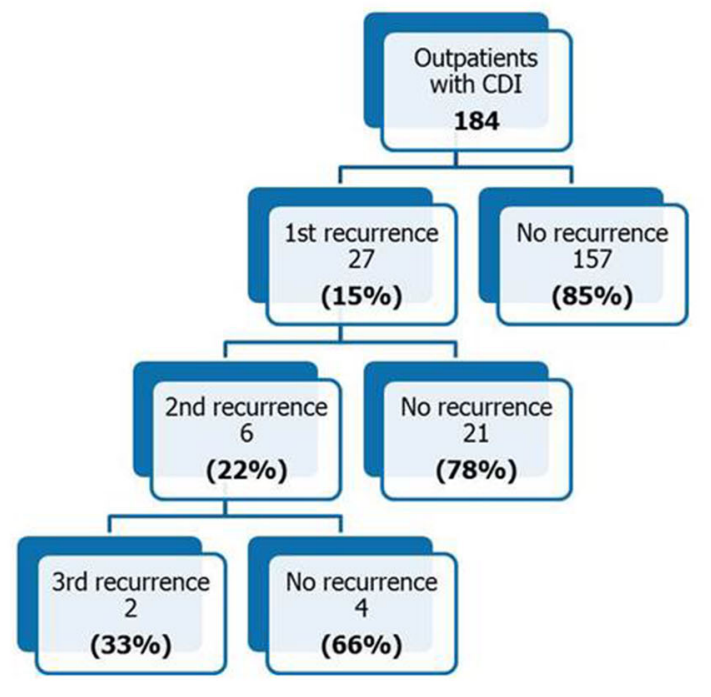

Inpatients with diagnosis of CDI

Primary diagnosis of CDI

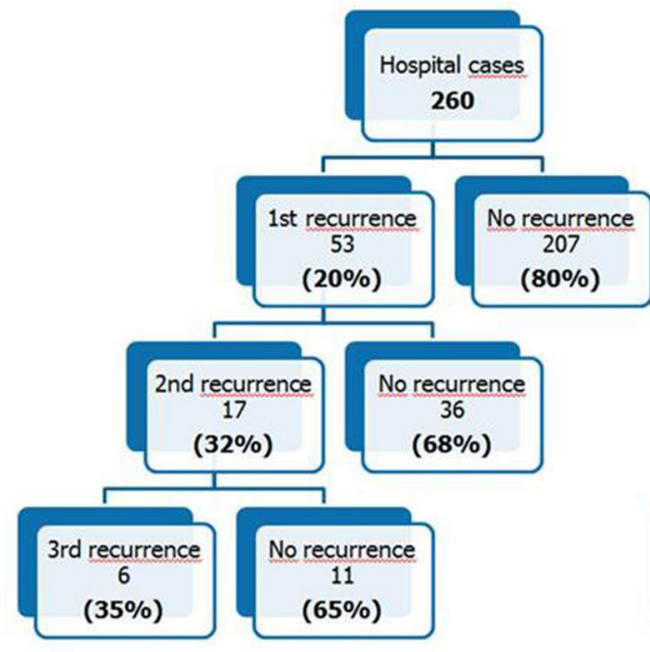

Secondary diagnosis of CDI

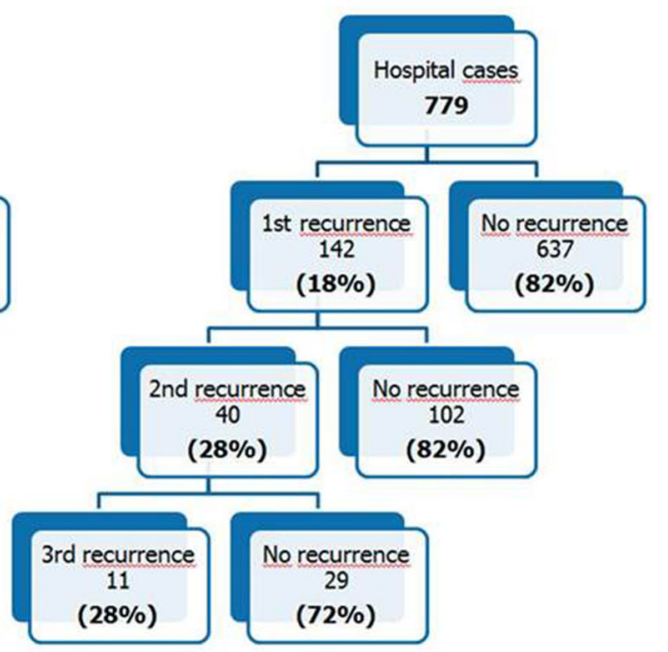

Fig. 3 Recurrence rates of patients with CDI in 2012

(Fig. 2), as previously pointed out in a European study by Bauer and colleagues [1]. According to their findings, $40 \%$ of all deaths may be attributed directly to CDI.

Our data include evaluation of antibiotic treatment characteristics which reflect that success rates of standard CDI therapies (metronidazole and vancomycin) are limited by (1) their non-guideline compliant use [26] with a high proportion of metronidazole therapy even for the second recurrence (43.5\%), and (2) their broad spectrum activity causing considerable perturbation of the intestinal microbiota [27]. Besides novel therapeutic strategies with antibiotics with a narrow spectrum of activity targeted strictly against $C$. difficile showing a diminished impact and damage on the anaerobic flora of the 


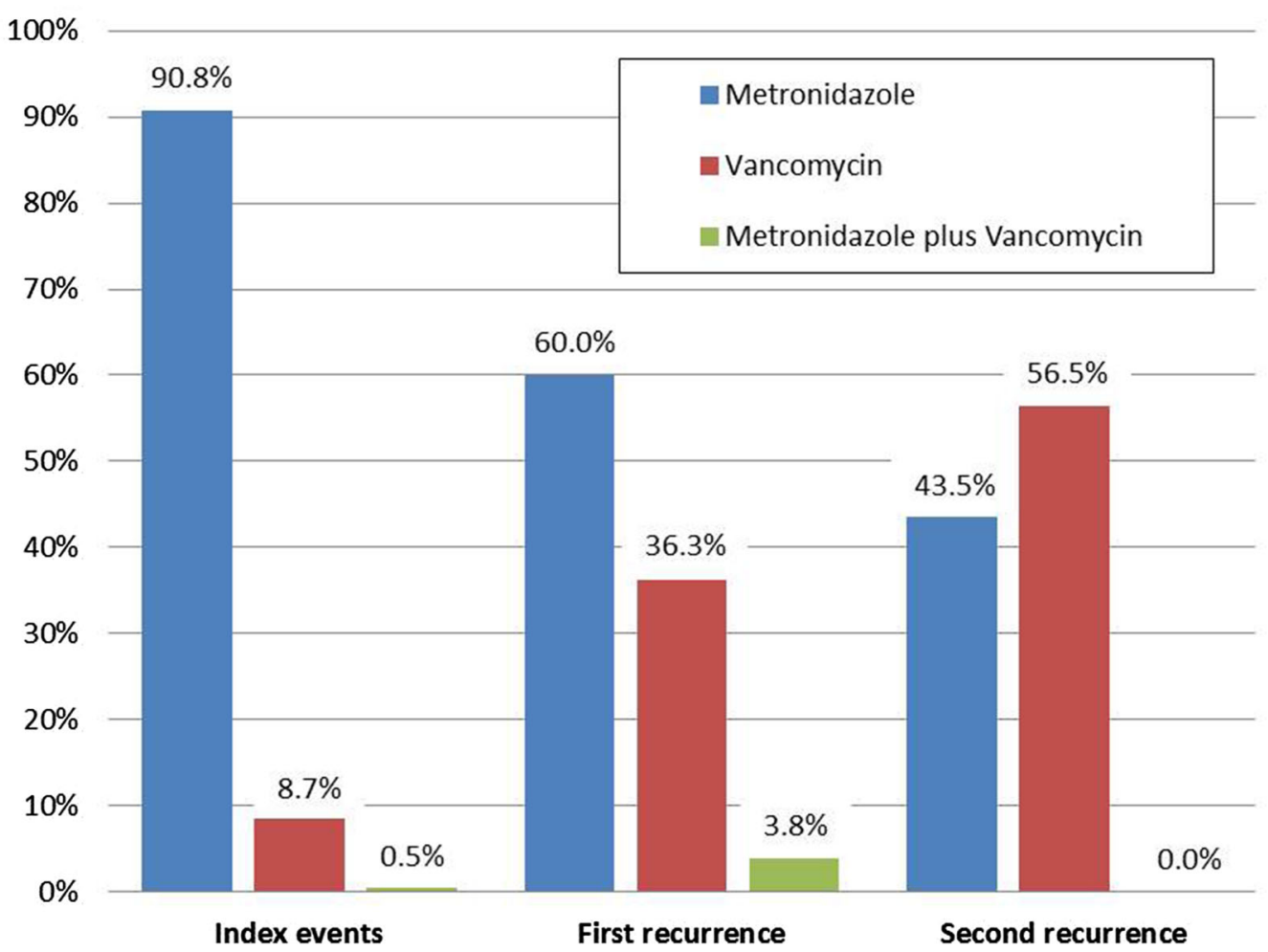

Fig. 4 Used antibiotic treatment regimens for CDI in outpatients in 2012

gastrointestinal tract such as fidaxomicin $[14,15,27]$, further innovative therapeutic strategies for CDI are urgently needed.

\section{Limitations}

Regarding interpretation of our results, the general limitations of a claims data-based analysis have to be considered. Our analysis largely depends on the quality of medical coding, which particularly relies on given clinical and diagnostic information, e.g., microbiological data and medication. Secondary data contain only information on services that are relevant for reimbursement. Coding of claims may be subject to incentives for reporting. Moreover, one has to consider that outpatient diagnoses of recurrent CDI without CDI-specific therapy within the same quarter could not be detected in the dataset. Inpatient diagnoses of recurrent CDI include only patients being discharged between the index date and first recurrence, or between first and second recurrence. Therefore, a recurrence of CDI within the same hospitalization period could not be tracked, which could have led to a false low recurrence rate in inpatients. To estimate the maximum number of recurrences within one hospital stay, the proportion of stays longer than 25 days was stated. Although recurrences were tracked starting from day 11 after the index date (assuming 10 days of standard therapy), a lack of differentiation regarding prolonged treatment of initial CDI and treatment of a genuine relapse remains. Regarding the assessment of mortality rates, our analysis is weakened by missing clinical information on the severity of CDI events. 


\section{CONCLUSION}

The reported incidence of CDI in Germany is higher than noted previously (83 cases per 100,000 population in 2012 according to this study covering the whole of Germany versus 14.8 cases per 100,000 population in 2006 in Saxony, Germany [24]). The recurrence rates of CDI increase with the number of relapses, but are lower in the analyzed database than reported in the literature $[17,18]$ despite the dominance of metronidazole treatment in outpatients. There is a strong need for novel targeted treatment options against recurrent CDI and preventive measures such as toxoid vaccines.

\section{ACKNOWLEDGMENTS}

Sponsorship for this study was funded by MSD Sharp \& Dohme GmbH, Haar, Germany. All named authors meet the International Committee of Medical Journal Editors (ICMJE) criteria for authorship for this manuscript, take responsibility for the integrity of the work as a whole, and have given final approval for the version to be published. We acknowledge support from the German Research Foundation (DFG) and the University of Leipzig within the program of Open Access Publishing.

Disclosures. C. Lübbert received financial support for consulting, lecture fees, and travel costs from Astellas, InfectoPharm, MSD, Novartis, Pfizer, and the Falk Foundation. L. Zimmermann and J. Borchert are employed by Leipziger Gesundheitsforen, Leipzig, Germany. B. Hörner is an employee of MSD Sharp \& Dohme GmbH, Haar, Germany. R. Mutters received financial support for consulting, lecture fees, and travel costs from Astellas, Basilea, InfectoPharm, MSD, Novartis, and Pfizer. A. C. Rodloff received financial support for consulting, lecture fees, and travel costs from AstraZeneca, BAG, Biorad, Gilead, InfectoPharm, MSD, Novartis, Pfizer, Roche, and ThermoFischer.

Compliance with Ethics Guidelines. This retrospective study was performed in accordance with the ethical guidelines of the 1964 Declaration of Helsinki and its later amendments. For this type of study formal consent is not required in accordance with the federal legislation of the Free State of Saxony, Germany. As the study did not modify patient management and the data were processed anonymously, the need for informed consent was waived.

Data Availability. The datasets analyzed during the current study are available from the corresponding author on reasonable request.

Open Access. This article is distributed under the terms of the Creative Commons Attribution-NonCommercial 4.0 International License (http://creativecommons.org/licenses/ by-nc/4.0/), which permits any noncommercial use, distribution, and reproduction in any medium, provided you give appropriate credit to the original author(s) and the source, provide a link to the Creative Commons license, and indicate if changes were made.

\section{REFERENCES}

1. Bauer MP, Notermans DW, van Benthem BH, et al. Clostridium difficile infection in Europe: a hospital-based survey. Lancet. 2011;377:63-73.

2. Leffler DA, Lamont JT. Clostridium difficile infection. N Engl J Med. 2015;372:1539-48. 
3. Lessa FC, Mu Y, Bamberg WM, et al. Burden of Clostridium difficile infection in the United States. N Engl J Med. 2015;372:825-34.

4. Jones M, Kuijper EJ, Wilcox MH. Clostridium difficile: a European perspective. J Infect. 2013;66:115-28.

5. Evans CT, Safdar N. Current trends in the epidemiology and outcomes of Clostridium difficile infection. Clin Infect Dis. 2015;60:S66-71.

6. Poutanen SM, Simor AE. Clostridium difficile-associated diarrhea in adults. CMAJ. 2004;171:51-8.

7. Freeman J, Bauer MP, Baines SD, Corver J, Fawley $\mathrm{WN}$, Goorhuis B, et al. The changing epidemiology of Clostridium difficile infections. Clin Microbiol Rev. 2010;23:529e49.

8. Jhung MA, Thompson AD, Killgore GE, et al. Toxinotype V Clostridium difficile in humans and food animals. Emerg Infect Dis. 2008;14:1039-45.

9. Debast SB, van Leengoed LA, Goorhuis A, et al. Clostridium difficile PCR ribotype 078 toxinotype V found in diarrhoeal pigs identical to isolates from affected humans. Environ Microbiol. 2009;11:505-11.

10. von Müller L, Mock M, Halfmann A, et al. Epidemiology of Clostridium difficile in Germany based on a single center long-term surveillance and German-wide genotyping of recent isolates provided to the advisory laboratory for diagnostic reasons. Int J Med Microbiol. 2015;305:807-13.

11. Pepin J, Valiquette L, Cossette B. Mortality attributable to nosocomial Clostridium difficile-associated disease during an epidemic caused by a hypervirulent strain in Quebec. CMAJ. 2005;173:1037-42.

12. Hensgens MP, Goorhuis A, Dekkers OM, et al. All-cause and disease-specific mortality in hospitalized patients with Clostridium difficile infection: a multicenter cohort study. Clin Infect Dis. 2013;56:1108-16.

13. Vardakas KZ, Polyzos KA, Patouni K, et al. Treatment failure and recurrence of Clostridium difficile infection following treatment with vancomycin or metronidazole: a systematic review of the evidence. Int $\mathrm{J}$ Antimicrob Agents. 2012;40:1-8.

14. Louie TJ, Miller MA, Mullane KM, et al. Fidaxomicin versus vancomycin for Clostridium difficile infection. N Engl J Med. 2011;364:422-31.

15. Cornely OA, Crook DW, Esposito R, et al. Fidaxomicin versus vancomycin for infection with
Clostridium difficile in Europe, Canada, and the USA: a doubleblind, non-inferiority, randomised controlled trial. Lancet Infect Dis. 2012;12:281-9.

16. Cornely OA, Miller MA, Louie TJ, et al. Treatment of first recurrence of Clostridium difficile infection: fidaxomicin versus vancomycin. Clin Infect Dis. 2012;55:S154-61.

17. Surawicz CM, Alexander J. Treatment of refractory and recurrent Clostridium difficile infection. Nat Rev Gastroenterol Hepatol. 2011;8:330-9.

18. Sheitoyan-Pesant C, Abou Chakra CN, Pepin J, et al. Clinical and healthcare burden of multiple recurrences of Clostridium difficile infection. Clin Infect Dis. 2016;62:574-80.

19. Vonberg RP, Reichardt C, Behnke M, et al. Costs of nosocomial Clostridium difficile-associated diarrhoea. J Hosp Infect. 2008;70:15-20.

20. Lipp MJ, Nero DC, Callahan MA. Impact of hospital-acquired Clostridium difficile. J Gastroenterol Hepatol. 2012;27:1733-7.

21. Wiegand PN, Nathwani D, Wilcox MH, et al. Clinical and economic burden of Clostridium difficile infection in Europe: a systematic review of healthcare-facility-acquired infection. J Hosp Infect. 2012;81:1-14.

22. Heimann SM, Vehreschild JJ, Cornely OA, et al. Economic burden of Clostridium difficile associated diarrhoea: a cost-of-illness study from a German tertiary care hospital. Infection. 2015;43:707-14.

23. Statistisches Bundesamt (Destatis). DRG-Statistik 2013-14. Vollstationäre Patientinnen und Patienten in Krankenhäusern (einschl. Sterbe- und Stundenfälle); ausgewählte Hauptdiagnose ICD-10 A04.7. Germany 2016. http://www.destatis.de. Accessed 7 Aug 2016.

24. Burckhardt F, Friedrich A, Beier D, Eckmanns T. Clostridium difficile surveillance trends, Saxony, Germany. Emerg Infect Dis. 2008;14:691-2.

25. Strausberg J. Epidemiology of Clostridium difficile infection. Dtsch Ärztebl Int. 2015;112:345.

26. Lübbert C, John E, von Müller L. Clostridium difficile infection: guideline-based diagnosis and treatment. Dtsch Arztebl Int. 2014;111:723-31.

27. Zhanel GG, Walkty AJ, Karlowsky JA. Fidaxomicin: a novel agent for the treatment of Clostridium difficile infection. Can J Infect Dis Med Microbiol. 2015;26:305-12. 